九州大学学術情報リポジトリ

Kyushu University Institutional Repository

\title{
PLANT ASSOCIATED ALTICINAE FROM THE BISMARCK RANGE, PAPUA NEW GUINEA (COLEOPTERA: CHRYSOMELIDAE)
}

Samuelson, G. A.

https://doi.org/10.5109/2449

出版情報 : ESAKIA. 21，pp.31-47，1984-02-15. Entomological Laboratory，Faculty of Agriculture， Kyushu University

バージョン:

権利関係 : 


\title{
PLANT ASSOCIATED ALTICINAE FROM THE BISMARCK RANGE, PAPUA NEW GUINEA (COLEOPTERA: CHRYSOMELIDAE)*
}

\author{
G. A. Samuelson \\ Department of Entomology, Bishop Museum, \\ Honolulu, Hawaii 96819, USA
}

\begin{abstract}
Nine species of plant associated Alticinae from the Mt Wilhelm area of the Bismarck Range, Papua New Guinea are treated. These include eight new species and one new genus: Typhodes aetherius n. gen. et n. sp., Manobiabismarckiana, cyatroxae, elysia, hirashimai, monilis n. spp., and Crepidodera piundaunde, stypheliae n. spp. Additional specimens of Setsaltica cyatheae Sam. are also treated. The new taxa are illustrated.
\end{abstract}

The Bismarck Range was one of the mountainous regions visited by the 1982 Hirashima Expedition. The high elevation, cultivated gardens near Keglsugl were sampled, as well as the relatively undisturbed montane forest, subalpine, and alpine ecosystems higher on the Mt Wilhelm massif. One of the places of special interest was the upper part of the Piunde-Aunde Lakes drainage, situated above $3000 \mathrm{~m}$ in a glacially cut valley. This area had been surveyed botanically (Wade \& McVean, 1969) and was occasionally visited by entomologists over the past decades. The Alticinae collected on the Hirashima Expedition and a part of the material collected on earlier visits to the area are reported upon now. Included are particularly important earlier collections made by the late J. Linsley Gressitt.

Piunde-Aunde Lakes. Confusion over the names of these two lakes makes it necessary to qualify the name of either lake as upper or lower lake in the lists of type material. Most specimen labels through the 1960s have Aunde as the lower lake and that is in agreement with Reiner (1960). Brass (1964) notes that there was confusion over the names of the lakes. The current series of topographical maps, Bundi quadrant, No. 7986 indicates the lower

\footnotetext{
* Contribution based on partial results of field work by Ministry of Education, Science and Culture (Japan) grant OSS-57041039 to Prof. Y. Hirashima and partial results of research and field work by National Science Foundation (USA) grant DEB-80-16438 to Bishop Museum. The publication of this work was also supported by MESC grant OSS58043039 to Prof. Hirashima, Kyushu University.
} 
lake as Piunde, and the Papua New Guinea Gazetteer (1974) also indicates the same by coordinates.

Types are deposited in Bishop Museum, Honolulu (BPBM) ; paratypes are distributed to the Australian National Insect Collection, Canberra City (ANIC) ; British Museum (Natural History), London (BMNH) ; Department of Primary Industry, Konedobu, PNG (KONE) ; Kyushu University, Fukuoka (KU) ; and New Zealand Arthropod Collection, DSIR, Auckland (NZAC).

\section{Typhodes, new genus}

Alticinae. Form subovate. Head somewhat retracted; labrum with anterior margin truncate ; frons triangular, elevated ; postantennal swellings triangular, swollen, slightly separated medially, with acute apices extending to interantennal space ; eye small, projecting; interocular space broad. Antenna exceeding elytral base ; outer flagellar segments more robust than inner ones; segment 7 slightly modified, with outer apical angle slightly produced. Prothorax with surface convex, lacking ante-basal impression. Elytron convex, humeri not raised ; puncturation confused; epipleuron horizontal. Ventral surfaces : thoracic sterna short ; procoxa globular-transverse, with intercoxal piece narrow but broader than a lamina; procoxal cavity broadly open behind; abdomen with sternum 1 as long or longer than 2-5 combined. Legs: metatibia flattened to feebly concave on retrotarsal surface; tibial spine simple ; tarsomere 3 bilobed ; ungues appendiculate. Wings vestigial. Abdomen with prepygidial terga membraneous. Spermatheca with receptacle bulbous, lacking receptacular process.

TyPe-species: Typhodes aetherius $\mathbf{n}$. gen. et $\mathbf{n}$. sp.

Name Derivation: Typhodes (Greek) =smoke, mist; gender masculine.

REMARKs: The new genus is most closely allied to Trachytetra Sharp from New Zealand, and it is distinguished from the type-species of the latter $[T$. rugulosa (Broun)] by having abdominal sternum 1 as long as or longer than the remaining sterna, instead of distinctly shorter. Typhodes is presumed to be relict in its distribution because of its isolation on high mountains in New Guinea and its close affinities with Trachytetra. Thus far, only one species of Typhodes is known, and it is represented only by material from the Bismarck Range.

\section{Typhodes aetherius, new species (Figs. 1A, 2A)}

Male (holotype). Form subovate, slightly tapering apically; dorsal surfaces rather strongly convex. Dorsum black, shining; elytron with slight lustre ; head, antenna, ventral surfaces, and legs piceous to black. Body length 2.3 $\mathrm{mm}$; breadth 1.55 . 
Head: frons triangular, surface turgid, anterior margin concave ; interantennal space convex below and concave above between apices of postantennal swellings, narrowest part of interantennal space about lx as broad as transverse diameter of antenna1 socket; orbit about $0.6 \times$ as broad as antenna1 socket; interocular space $1.68 \times$ as broad as maximum diameter of eye; eye longer than broad, somewhat narrowed below; gena about $0.75 \times$ as deep as eye; postantennal swellings \pm smooth, surfaces swollen ; vertex smooth, shining. Antenna attaining middle of elytron ; scape swollen apically ; pedicel swollen but narrower than scape; 3 gradually thickened to apex, slightly longer than pedicel or 4; 4-5 thickened apically, subequal in length; 7 more robust, with outer apical angle produced; 8-10 robust, slightly longer than 4-6; 11 robust with apical half narrowed to acute apex; outer flagellar segments more densely clothed with pubescence than inner ones. Pronotum about $0.65 \times$ as long as broad, broadest near middle; pronotal base slightly narrower than basal breadth of elytra ; anterior angle oblique-rounded; side, posterior angle, and base convex; disc evenly convex, surface smooth; discal punctures moderately deep and mostly $0.7 \times$ as broad as interspaces ; interspaces flat, smooth, sparsely micropunctate. Elytron about $2.4 \times$ as long as broad, broadest near middle ; humeral angle rounded; apical angle square-briefly rounded ; surface convex; central discal punctures mostly $0.7-1$ x as large as interspaces; interspaces flat, shining, sparsely micropunctate ; epipleuron broadest along basal 0.25 , thence narrowed and continued to preapex. Ventral surfaces: metasternum smooth, shining medially ; abdomen relatively dull, granulate-punctate; sternum 1 about 1.5 as long as 2-5. Legs: tibiae \pm stout, gradually thickened towards apices; metafemur not quite half as broad as long, surface granulate, punctate apically ; lengths of metafemur, -tibia, -tarsus in $\mathrm{mm}$ are 0.80: 0.62: 0.52; basitarsus not quite as long as remainder. Aedeagus (paratype) as figured.

Female (allotype) . Castaneous overall. Dorsal puncturation closer than in male, with punctures commonly $1 \mathrm{x}$ as large as interspaces on pronotal and elytral discs. Antenna1 segment 7 modified as in male. Body length $2.65 \mathrm{~mm}$; breadth 1.6 .

Type materias: PAPUA NEW GUINEA : Holotype male, allotype female, 10 paratopotypes, NW of Lake Piunde [as upper lake], 3600-3800 m, 13. VI. 1967, Coprosma papuensis, G. A. Samuelson; paratopotypes : 9, same data as holotype but on Stypheliasuaveolens; 2, Mt Wilhelm [vicinity of Piunde-Aunde Lakes], 3600 m, VI. 1955, J. L. Gressitt; 8, same loc., 3750-3950 m, V. 1966, Gressitt ; 21, same loc., 3560-4200 m, VIII. 1969, Ericaceae or Rhododendron, Gressitt ; 1, same data but $3560 \mathrm{~m}$, on Styphelia leucopogon; 23, same data but 3560-4400 m, on Coprosma; 10, same data but 3660-4000 m, without host; 1, near Lake Aunde [as lower lake], 3600 m, VII. 1955, Gressitt; 20, same loc., 34003500 m, VII. 1963, J. Sedlacek ; 12, same loc., 3350 m, VI. 1967, Samuelson; 4, 
same data but on Vaccinium creuentum; 8, same data but $3400-3550 \mathrm{~m}$, on Dimorphanthera microphylla; 3, same loc., VIII. 1968, R. C. A. Rice; 1, Lake Piunde [as lower lake], 3560 m, VIII. 1982, Samuelson.

Holotype, allotype, many paratypes deposited in BPBM (type no. 13048). Paratypes distributed to ANIC, BMNH, KONE, KU, and NZAC.

Remarks: The new species resembles Trackytetra robusta Broun [New Zealand] because of its convex body form; however, the presence of a spermathecal receptacular process (see Samuelson, 1973: 6) in robusta indicates that robusta is more closely related to Linaltica Samuelson [Fiji, Tonga] than it is to Trackytetra nugulosa or the new species.

Adults were collected from five genera of plants of three families :Epacridaceae (Styphelia), Ericaceae (Dimorphanthera, Rhododendron, Vaccinium), and Rubiaceae (Coprosma). The larval host(s) are not known. The altitudinal range for the beetle is fairly broad at 3350 to $4400 \mathrm{~m}$; however, the lower limit (grassland-shrub formation) is still a cool, subalpine environment. The highest-collected beetles taken from plants were from Coprosma at $4400 \mathrm{~m}$.

\section{Setsaltica cyatheae Samuelson}

Setsaltica cyatheae Sam., 1971, Pacific Ins. 13(3-4): 514.

Specimens examined : PAPUA NEW GUINEA :2, near Lake Aunde [as lower lake], $3550 \mathrm{~m}, 12$. VI. 1967, Cyatkea muelleri, G. A. Samuelson; 1, lower Piunde-Aunde Vall., 3300 m, 15. VI. 1967, ferns and tree ferns, Samuelson.

Remarks: This species is closely associated with its tree fern host, Cyatkea muelleri. The specimen without precise host data was not taken from Cyatkea atrox, the predominant tree fern at around $3300 \mathrm{~m}$; it was generally swept from various other pteridophytes not identified further.

Manobia bismarckiana, new species (Figs. 1B, 2B)

Male (holotype). Body form subrobust; prothorax narrow relative to elytral breadth. Dorsum black, shining, with bright slightly golden lustre most pronounced on elytra; antenna orange-testaceous, apical 2 segments somewhat darker, pitchy; ventral surfaces black ; legs orange-testaceous, metafemur somewhat darker, pitchy. Body length $2.45 \mathrm{~mm}$; breadth 1.3 .

Head: frons rather long, gently convex anteriorly, weakly carinate medially above; interantennal space concave overall but briefly convex medially, about $1.65 \times$ as broad as transverse diameter of antenna1 socket; orbit subequal in breadth to antenna1 socket; interocular space barely narrowest below, $1.46 \times$ as broad as greatest diameter of eye; gena about $0.8 \times$ as deep as eye; postantennal swellings small, \pm short-oblique, briefly swollen, and delimited from vertex by deep oblique groove; vertex smooth, impunctate, surface convex. Antenna extending to nearly middle of elytron; inner flagellar seg- 
ments fairly slender, gradually thickened toward apices; apical 5 segments longer, heavier than preceding ones. Prothorax $0.92 \times$ as long as broad, broadest at anterior angles, thence narrowed to prebasal constriction; constriction about $0.58 \times$ as broad as elytra at humeri ; anterior angle oblique-rounded; side sinuate, convex anteriorly to concave basally; posterior angle produced; base bisinuate, median lobe weakly produced; disc quite convex at middle, broadened and less strongly convex anteriorly; discal punctures deep, commonly $2-3 \times$ as large as interspaces; punctures larger anterolaterally and prebasally ; interspaces mostly flat; ante-basal impression sinuate, moderately deep, punctate. Elytron $2.8 \times$ as long as broad, broadest behind middle, side gently convex, thence obliquely narrowed to apex; epipleuron finely punctulate externally, otherwise smooth and continued to apex; humerus moderately strongly produced; basal disc moderately swollen, thence moderately deeply and transversely depressed; punctures largest in and following depression ; puncture rows $4+5$ anastomosed behind depression; central discal punctures mostly $1-1.5 \times$ as large as interstices and $2-3 \times$ as large as transverse interspaces ; punctures well impressed apically ; interstices quite convex, smooth. Ventral surfaces : propleuron smooth, impunctate ; metasternum smooth, sparsely punctulate; abdomen alutaceous to subgranulate. Legs: metafemur $2.8 \mathrm{x}$ as long as broad, surface alutaceous, subgranulate apically ; relative lengths of metafemur, -tibia, -tarsus in $\mathrm{mm}$ are 0.84: $0.82: 0.56$; basitarsus not as long as remainder. Wing fully developed. Aedeagus (paratype) as figured.

Female (allotype). Similar to male. Body length $2.65 \mathrm{~mm}$; breadth 1.4 .

Type material: PAPUA NEW GUINEA: Holotype male, allotype female, many paratopotypes, Mt Wilhelm [vicinity of Piunde-Aunde Lakes], $3560 \mathrm{~m}, 1$. VIII. 1969, Urticaceae, \#6618, J. L. Gressitt; paratopotypes: 1 , same data but 3, 7. VIII, on Styphelia leucopogon; 2, same loc., 3660 m, VII. 1977, Urticaceae, N. Nadkarni; many, same loc., 3600 m, VII. 1955, Gressitt; 6, same loc., Lake Aunde [as lower lake], 3600 m, VII. 1955, Gressitt; 1, same loc., Lake Aunde [as lower lake], V. 1968, R. C. A. Rice; 1, same loc., 3550 m, VIII. 1969, Rosaceae, Gressitt ; 2, same loc., 3300 m, VI. 1967, tree ferns and ferns, G. A. Samuelson ; paratypes : 1, Mt Wilhelm [well below lakes], 2750 m, VIII. 1969, Gressitt ; 1, same loc., 2800-2900 m, VII. 1963, J. Sedlacek ; 4, same loc., 3000 m, VI-VII. 1955, Gressitt.

Holotype, allotype, many paratypes deposited in BPBM (type no. 13049). Paratypes distributed to ANIC, BMNH, KONE, KU, and NZAC.

REMARKS: The new species falls into a grouping of glabrous species of Liprus which will have to be reassigned generically. The new species differs from Liprus rugosus Bryant [New Guinea : Mt Tafa] by larger body size (length ca. $2.5 \mathrm{~mm}$ instead of 2.0) and by proportionally smaller eyes and longer genae; rugosus also lacks the slightly golden lustre of the dorsum and has darker antennae and legs than the new species. 
This appears to be the first conclusive record of Manobia being associated with Urticaceae in New Guinea. The larval host is not yet known. The few records from other plants, Rosaceae, Styphelia, tree ferns and ferns, are probably incidental.

\section{Manobia cyatroxae, new species (Figs. 1C, 2C)}

Male (holotype). Body form subrobust, prothorax narrow relative to elytral breadth. Dorsum yellow to orange-testaceous; head and pronotum slightly darker than elytra but elytral punctures showing through cuticle as darker pitchy spots; antenna with segments 1-7 yellow-testaceous, 8-11 fuscous; ventral surfaces orange-testaceous ; legs yellow-testaceous. Body length $2.15 \mathrm{~mm}$; breadth 1.15 .

Head: frons rather evenly convex transversely, surface smooth; interantennal space concave between raised margins of antenna1 sockets, breadth subequal to transverse diameter of antenna1 socket; orbit about $0.65 \times$ as broad as antenna1 socket; interocular space 1.14 as broad as greatest diameter of eye; gena about $0.75 \times$ as deep as eye ; postantennal swellings narrow, obsolescent; postantennal area delimited from vertex by fine oblique line; vertex evenly convex, smooth-impunctate. Antenna extending almost to middle of elytron ; inner flagellar segments slender, gradually thickened to apices, 4 outer segments turgid, thicker than inner ones. Prothorax $0.89 x$ as long as broad, broadest at anterior angles, narrowest prebasally, with constriction about $0.61 \mathrm{x}$ as broad as elytra at humeri; anterior angle short, oblique-rounded; base bisinuate, median lobe fairly deep; disc closely punctate with interspaces smooth, punctures commonly $3-4 \times$ as large as interspaces ; prebasal area \pm flattened-feebly concave; prebasal punctures similar to discal ones. Elytron about 2.9 as long as broad, broadest slightly behind middle ;epipleuron punctate, ending preapically ; humerus moderately produced; basal area submoderately swollen, thence transversely depressed ; interstices not interrupted in postbasal depression ; disc convex, somewhat inflated apically; central discal punctures large, shallow, mostly about $2 \mathrm{x}$ as large as interstices and 5 $\mathrm{x}$ as large as transverse interspaces; interstices smooth, quite swollen, almost costate in appearance. Ventral surfaces: propleuron smooth, moderately punctate; metasternum smooth, turgid, bearing a series of deep punctures along anterior margin ; abdominal sterna smooth-alutaceous, \pm sparsely punctate. Legs: metafemur about $2.9 \times$ as long as broad, surface \pm smooth ; relative lengths of metafemur, -tibia, -tarsus in $\mathrm{mm}$ are 0.70: 0.64: 0.44; basitarsus not quite as long as remainder. Wing fully developed. Aedeagus (paratype) as figured.

Female (allotype). Similar to male. Body surfaces and legs mostly yellowtestaceous, elytral punctures not showing through as darker spots; elytron 
more inflated apically than in male; antenna not quite attaining middle of elytron; abdominal sternum 1 with a series of large punctures along anterior margin. Body length $2.4 \mathrm{~mm}$; breadth 1.3 .

Type material: PAPUA NEW GUINEA : Holotype male, allotype female, many paratopotypes, lower Piunde-Aunde Vall. [Kombuglmambuno], $3300 \mathrm{~m}$, 15. VI. 1967, Cyatkea atrox, G. A. Samuelson; 13 paratopotypes, same loc., 3200 m, 31. VII. 1982, Cyatkea atrox, Samuelson ; 3, same data but without host; paratypes: 2, Mt Wilhelm [vicinity of Piunde-Aunde Lakes], 3600 m, VI. 1955, J. L. Gressitt; 1, same data but VII. 1955; 1, same loc., 3000 m, VII. 1955, Gressitt.

Holotype, allotype, most paratypes deposited in BPBM (type no. 13050). Paratypes distributed to ANIC, BMNH, KONE, KU, and NZAC.

REMARKS: The new species differs from Manobia papuana Bryant [New Guinea: Mt Tafa] by having the prothorax nearly parallel-sided instead of moderately narrowed toward preapex; pronotal disc more closely and deeply punctate (punctures $3-4 \times$ instead of $0.2-0.3 \mathrm{x}$ as large as interspaces) ; and elytral disc with inner interstices convex instead of flat.

Adults of the new species were taken almost exclusively from foliage of the tree fern, Cyatkea atrox. Larvae were not collected. The host is the predominant tree fern at the lower end of the Piunde-Aunde Lakes grassland.

\section{Manobia elysia, new species (Figs. 1D, 2D)}

Female (holotype). Body form subrobust ; prothorax somewhat narrow relative to elytral breadth. Dorsum pitchy orange-fuscous, elytron with pale yellow-testaceous areas as follows : subquadrate spots preceding and following postbasal depression and entire apical 0.25 ; antenna orange-testaceous, apical 3 segments fuscous; ventral surfaces dark red-fuscous; legs pitchy orangefuscous. Length $2.15 \mathrm{~mm}$; breadth 1.15 .

Head: frons short, very finely carinate medially above ; interantennal space concave, about $1.65 \sim$ as broad as transverse diameter of antenna 1 socket; orbit about $0.35 \times$ as broad as antenna1 socket; interocular space narrowest below, $1.09 \mathrm{x}$ as broad as greatest diameter of eye ; gena about $0.35 \sim$ as deep as eye; postantennal swellings subquadrate-oblique, with upper inner angles closest, surface quite swollen, smooth, and delimited from vertex by deep oblique-sinuate groove ; vertex briefly swollen at anterior extremity, disc smooth, impunctate, evenly convex. Antenna not quite reaching middle of elytron; flagellar segments \pm slender, apical 3 segments distinctly thicker than preceding ones. Prothorax 0.72 as long as broad, broadest at posterior angles and \pm middle; prebasal area $0.67 \sim$ as broad as elytra at humeri ; anterior angle oblique-rounded ; side convex ; posterior angle produced ; base bisinuate, median lobe well produced; disc rather closely punctate with interspaces smooth; punctures deep and commonly $2-3 \times$ as large as interspaces 
but disc briefly impunctate medially for a short distance ; ante-basal impression sinuate, moderately deep ; median prebasal punctures distinctly larger than others. Elytron 2.8 as long as broad, broadest behind middle, thence obliquely and gradually narrowed to apex; epipleuron finely punctate externally, otherwise smooth and ending preapically; humerus submoderately produced; basal disc moderately swollen, thence moderately and transversely depressed; punctures of depression largest; central discal punctures mostly $0.7 \times$ as large as interstices and $3 \times$ as large as transverse interspaces; punctures well impressed apically ; interstices quite swollen, smooth. Ventral surfaces :propleuron smooth, impunctate; metasternum broadly swollen, smooth, sparsely punctulate; abdominal sterna alutaceous to subgranulate, moderately punctulate. Legs: metafemur $2.5 \times$ as long as broad; surface mostly smooth but abruptly granulate apically ; relative lengths of metafemur, -tibia, -tarsus in $\mathrm{mm}$ are $0.66: 0.58: 0.42$; basitarsus not quite as long as remainder. Wing fully developed.

Male (allotype). Similar to female. Ground color of elytron paler fuscous than in female but pale testaceous areas still evident; antenna extending to about middle of elytron. Aedeagus (paratype) as figured. Body length $1.9 \mathrm{~mm}$; breadth 1.0 .

Type material : PAPUA NEW GUINEA : Holotype female, Mt Wilhelm [vicinity of Piunde-Aunde Lakes], 3560 m, 1. VIII. 1969, Urticaceae, \#6618, J. L. Gressitt; allotype male, same loc., $3600 \mathrm{~m}$, 2. VII. 1955, Gressitt; paratopotypes: 1, same data as holotype but 7. VIII. 1969, on Olearia; 3, same loc., $3600 \mathrm{~m}$, VII. 1955, Gressitt; 1, lower Piunde-Aunde Vall. [Kombuglmambuno], 3300 m, VI. 1967, tree ferns and ferns, G. A. Samuelson; 1, same data but without host.

Holotype, allotype, most paratypes deposited in BPBM (type no. 13051). Paratypes distributed to BMNH and KONE.

Remarks: The new species differs from Manobia cyatroxae, n. sp. by having prothorax shorter, stouter (ca. $0.7 \times$ instead of $0.9 \times$ as long as broad) ; prothorax also broadest posteriorly instead of anteriorly ; ante-basal impression of pronotum well impressed instead of barely impressed; and by generally darker coloration of dorsum.

The small number of plant associates for adults includes Compositae (Olearia), Pteridophyta (not identified to family), and Urticaceae. The definitive host is still unknown.

\section{Manobia hirashimai, new species (Figs. 1E,2E)}

Male (holotype). Body form subelongate, prothorax fairly broad relative to elytral breadth. Dorsum black, shining; antenna with segments 1-6 yellowtestaceous but 1, 5-6 somewhat pitchy, 7-11 orange-fuscous; ventral surfaces and metafemur black, shining; other femora and tibiae largely dark but basal 

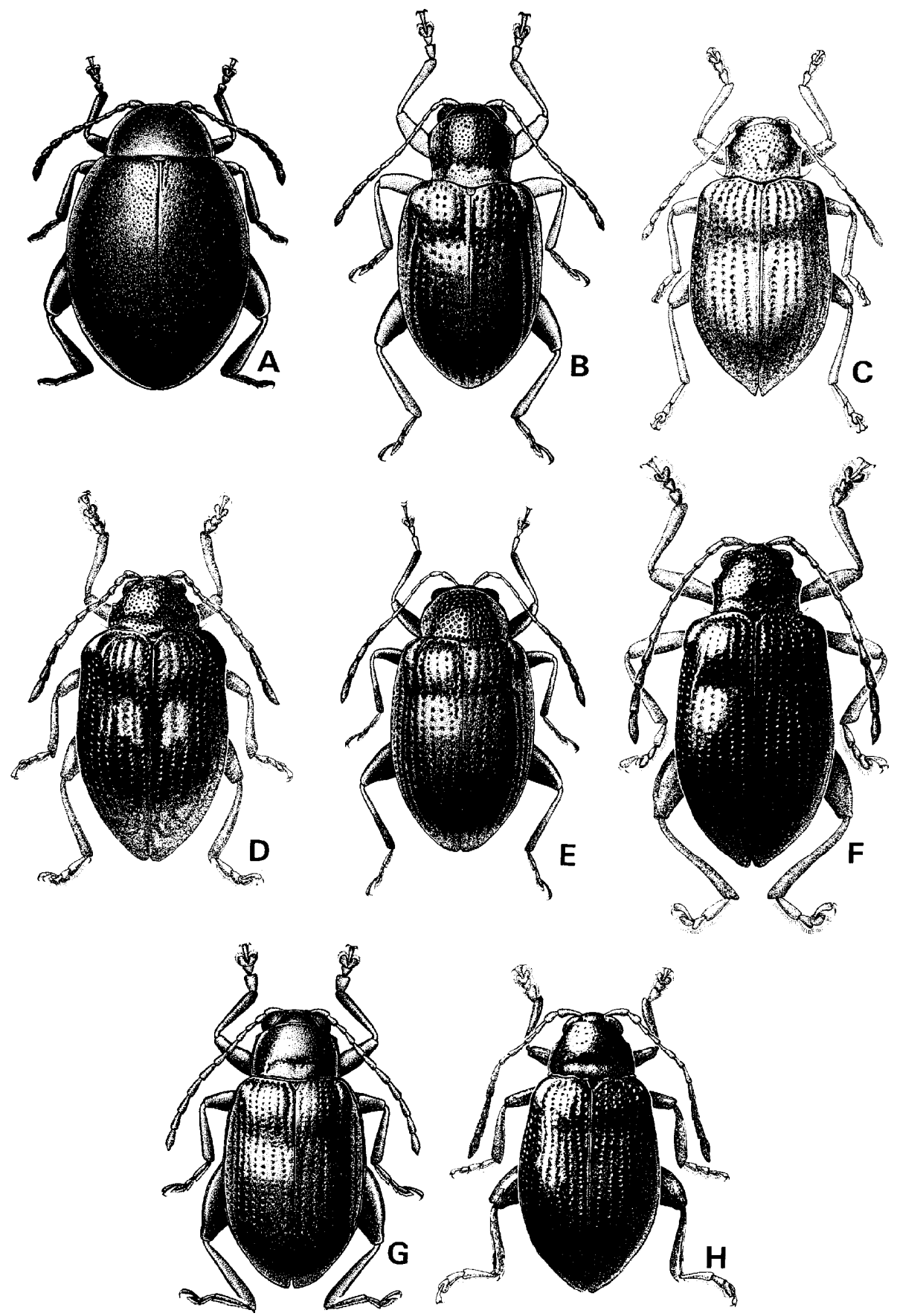

Fig. 1. Dorsal view. A :Typhodes aetherius n. gen. et n. sp., B: Manobia bismarckiana n. sp. ;

C: M. cyatroxae n. sp. ; D: $M$. elysia n. sp. ; E: M. hirashimai n. sp. ; F : M. monilis n. sp. ; $\mathrm{G}$ : Crepidodera piundaunde n. sp. ; H: C. stypheliae n. sp. 
0.3 of pro- and mesotibia paler, orange-fuscous; tarsi orange-testaceous. Body length $2.0 \mathrm{~mm}$; breadth 1.05 .

Head: frons transversely convex below, briefly and very finely carinate medially above; interantennal space concave between raised margins of antennal sockets, breadth about $1.2 \times$ as broad as transverse diameter of antennal socket; orbit about $0.8 \times$ as broad as antenna1 socket ; interocular space $1.05 \times$ as broad as greatest diameter of eye ; gena about $0.55 \mathrm{x}$ as deep as eye ; postantennal swellings \pm subquadrate-oblique, swollen, smooth, closest at their upper inner angles, and delimited from vertex by deep oblique groove; vertex evenly convex, smooth, impunctate. Antenna extending to about middle of elytron; inner flagellar segments slender, \pm gradually thickened to apices; outer 5 segments distinctly longer and heavier than preceding ones. Prothorax $0.77 \times$ as long as broad, broadest at posterior angles, narrowest just behind anterior angles; prebasal area about $0.73 \times$ as broad as elytra at humeri; anterior angle oblique ; side weakly convex anteriorly, be-
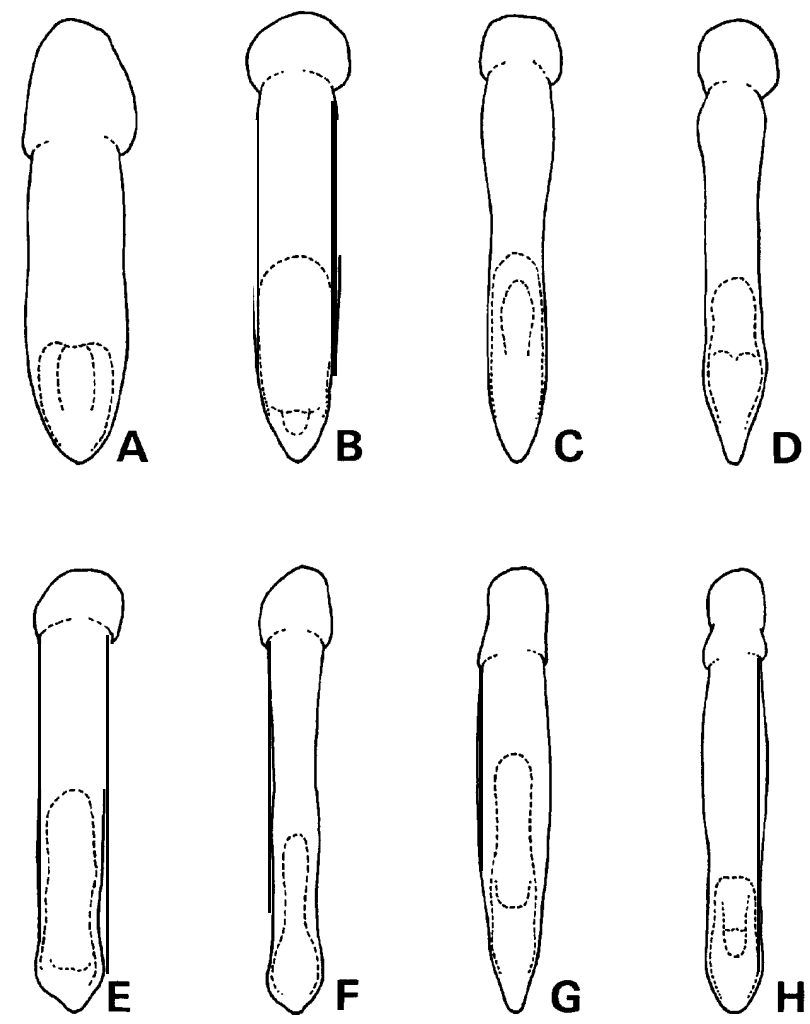

Fig. 2. Aedeagus, dorsal view. A: Typhodes aetherius n. gen. et n. sp. ; B: Manobia bismarckiana n. sp. ; $C: M$. cyatroxae n. sp. ; D: $M$. elysia n. sp.; E :M. hirashimai n. sp. ;F:M monilis n. sp.; G : Crepidodera piundaunde n. sp.; H : C. stypheliae n. sp. 
coming straight and narrowed toward base ; posterior angle produced; base bisinuate, median lobe well produced; disc deeply punctate, punctures large and commonly $2-3 \times$ as large as interspaces ; interspaces smooth, convex, shining; prebasal area somewhat flattened but still convex ; prebasal punctures similar to discal ones. Elytron $3 \times$ as long as broad, side gently convex along middle, thence convexly narrowed to apex; epipleuron convex, punctate along outer margin, otherwise smooth and ending preapically; humerus moderately well produced; basal area submoderately swollen, thence rather shallowly and transversely depressed ; punctures of depression mostly larger and somewhat confused behind humeral swelling; central discal punctures moderately large, deep, commonly $1 \mathbf{x}$ as large as interstices and $3 \mathrm{x}$ as large as transverse interspaces ; punctures well impressed apically ; interstices smooth, quite swollen. Ventral surfaces: propleuron smooth, shining; metasternum swollen, smooth, sparsely punctulate and with a series of deep punctures along anterior margin; abdominal sterna alutaceous to subgranulate. Legs: metafemur 2.4 as long as broad, largely smooth, subgranulate apically; relative lengths of metafemur, -tibia, -tarsus in mm are $0.68: 0.62: 0.35$; basitarsus about as long as remainder. Wing fully developed. Aedeagus (paratype) as figured.

Female (allotype). Similar to male. Apices of all femora and bases of all tibiae pale, orange-fuscous. Body length $1.85 \mathrm{~mm}$; breadth 1.0.

Type materias: PAPUA NEW GUINEA: Holotype male, 2 paratopotypes, lower Piunde-Aunde Vall. [Kombuglmambuno], 3300 m, 15. VI. 1967, G. A. Samuelson ; allotype female, same loc., 3000 m, VI. 1955, J. L. Gressitt; 3 paratopotypes, same data as holotype but on tree ferns and ferns; paratypes: 1, same data as allotype but VII. 1955; 1, Lake Aunde [as lower lake], V. 1968, R. C. A. Rice.

Holotype, allotype, most paratypes deposited in (type no. 13052). Paratype deposited in BMNH.

Remarks: The new species differs from Manobia cyatroxae, n. sp. by having prothorax shorter, stouter (ca. $0.8 \times$ instead of $0.9 \times$ as long as broad) and broadest basally instead of apically ; lacking pronotal ante-basal impression instead of weakly impressed; and by having dorsum black instead of pale.

The only plant associate reported for the new species is a pteridophyte, without further identification.

\section{Manobia monilis, new species (Figs. 1F, 2F)}

Male (holotype). Body form subelongate, prothorax narrow relative to elytral breadth. Dorsum dark fusco-castaneous to piceous, shining; antenna with segments 1-6 yellow-testaceous, 7-11 fuscous; ventral surfaces and metafemur dark fuscous, legs otherwise yellow-testaceous. Body length $2.15 \mathrm{~mm}$; 
breadth 1.05 .

Head: frons feebly raised medially, surface smooth, shining; interantennal space concave, about $1.3 \sim$ as broad as transverse diameter of antenna 1 socket; orbit about $0.5 \times$ as broad as antenna1 socket; interocular space narrowest above and below middle, $1.08 \times$ as broad as greatest diameter of eye; gena about $0.5 \times$ as deep as eye; postantennal swellings subquadrate, slightly swollen, smooth, separated medially with lower angles more distant than upper angles ; vertex smooth, impunctate, shining, surface evenly convex. Antenna extending just to apical 0.33 of elytron; flagellar segments slender, gradually thickened to apices, 3 apical segments slightly broader than preceding ones. Prothorax $0.87 \times$ as long as broad, broadest at anterior angles, narrowest just before posterior angles, with constriction about $0.60 \times$ as broad as elytra at humeri; anterior angle oblique, moderately short ; side rather straight and gradually narrowed toward base; base bisinuate, median lobe moderately produced ; disc smooth with deep punctures mostly about 0.7 $\times$ as large as interspaces; ante-basal impression sinuate, rather briefly and deeply impressed, impression with punctures; prebasal area with punctures distinctly larger than discal ones. Elytron about $3 \times$ as long as broad, broadest near middle, side gently convex along middle, thence more gradually and obliquely narrowed apically ; epipleuron with fine marginal punctures, otherwise smooth, impunctate and continued to preapex; humerus moderately well produced; disc basally swollen, thence transversely and moderately depressed; interstices not interrupted in depression, except that puncture rows $5+6$ anastomosed postbasally and beginning in depression; punctures of depression distinctly larger than others; central discal punctures moderately deep and commonly $0.3-0.5 \times$ as large as interstices and $0.5 \times$ as large as transverse interspaces ; punctures smaller to obsolete apically ; interstices flat, smooth, shining. Ventral surfaces : propleuron smooth, shining; metasternum turgid, smooth, with a series of large punctures along anterior margin; abdominal sterna smooth, sparsely punctate. Legs: metafemur about $3 \mathrm{x}$ as long as broad, surface \pm smooth, subgranulate apically ; relative lengths of metafemur, -tibia, -tarsus in $\mathrm{mm}$ are $0.80: 0.74: 0.46$; basitarsus not quite as long as remainder. Wing fully developed. Aedeagus (paratype) as figured.

Female (allotype). Similar to male. Antenna extending to about middle of elytron. Body length $2.5 \mathrm{~mm}$; breadth 1.25 .

Type MATERIAL: PAPUA NEW GUINEA : Holotype male, Kombuglmambuno, above Keglsugl [lower Piunde-Aunde Vall. grassland], $3200 \mathrm{~m}, 31$. VII. 1982, Cyathea atrox, G. A. Samuelson; allotype female, same loc., 3300 m, 15. VI. 1967. various ferns and tree ferns, Samuelson; paratopotypes: 8, same data as holotype but 1 lacking host data; 10, same data as allotype but 3 lacking host data ; 3, same data as allotype but on Cyathea atrox; paratypes: 1, same loc., 3000 m, VI. 1955, J. L. Gressitt; 2, same loc., 2600-3600 m, VIII. 1969, Gressitt. 
Holotype, allotype, most paratypes deposited in BPBM (type no. 13053). Paratypes distributed to ANIC, BMNH, KONE, KU, and NZAC.

$\mathrm{REMARKS}$ : The new species differs from Manobia pallidicornis Jacoby $[\mathrm{New}$ Guinea] by being distinctly larger (length ca. 2.15-2.5 mm instead of 1.5-1.7 $\mathrm{mm}$ ) ; eyes relative small, distinctly smaller than interocular space instead of larger ; eyes also much less protruding; and pronotal disc with punctures distinct instead of nearly impunctate.

Adults were collected from Cyathea atrox, and various ferns and other tree ferns from the lower end of the Piunde-Aunde Lakes grassland.

\section{Crepidodera piundaunde, new species (Figs. 1G,2G)}

Male (holotype). Form subelongate, gently convex along middle, evenly and convexly narrowed to apex. Dorsum black with dark metallic green lustre ; antenna with segments 1-4 orange-testaceous, 5-11 fuscous ; ventral surfaces and legs mostly dark fuscous to piceous. Body length $2.5 \mathrm{~mm}$; breadth 1.35 .

Head: frons raised medially, depressed laterally ; interantennal space convex medially and briefly impressed at side, breadth about $1.3 \times$ as broad as transverse diameter of antenna 1 socket; orbit about $0.85 \times$ as broad as antennal socket; interocular space narrowest above, $1.14 \times$ as broad as greatest diameter of eye; gena about $0.5 \times$ as deep as eye; postantennal swellings narrow-oblique, raised, smooth, and delimited from vertex by oblique groove extending below into interantennal space ; vertex slightly roughened-alutaceous anteriorly and becoming smoother and more evenly convex posteriorly. Antenna extending to middle of elytron; inner flagellar segments gradually thickened to apices, outer segments slightly turgid. Prothorax 0.69 as long as broad, broadest behind middle and distinctly narrower than elytra at humeri ; anterior angle short, oblique, slightly produced; side feebly convex, nearly straight along middle; base sinuate, broadly but weakly convex medially; disc granulate-punctate ; discal punctures \pm deep and commonly $0.5 \times$ as large as interstices ; ante-basal impression sinuate, distinctly impressed across middle. Elytron about $2.7 \times$ as long as broad, broadest along middle; humerus moderately raised; disc slightly raised basally, thence shallowly and gradually depressed postbasally ; central discal punctures moderately deep, mostly 0.7-1 x as large as interstices and $2 \times$ as large as transverse interspaces ; interstices slightly raised, convex ; strial punctures well impressed apically. Ventral surfaces : propleuron smooth, shining; metasternum turgid, smooth-alutaceous, shining medially ; abdominal sterna granulate ; sternum 5 with very deep subcircular depression apically, preceded by fine median line. Legs: metafemur about $2.2 \times$ as long as broad, mostly smooth-alutaceous, becoming \pm granulate apically; lengths of metafemur, -tibia, -tarsus in mm are $0.98: 0.72: 0.64$; 
hasitarsus gradually broadened to apex, about as long as remainder. Aedeagus (paratype) as figured.

Female (allotype). Similar to male. Sternum 5 nearly flattened, surface granulate, with fine median line slightly broadened and deepened at apex ; metabasitarsus slender. Body length $2.75 \mathrm{~mm}$; breadth 1.4.

Type material: PAPUA NEW GUINEA : Holotype male, allotype female, 11 paratopotypes, Mt Wilhelm [vicinity of Piunde-Aunde Lakes], 3560 m, 3, 7. VIII. 1969, Styphelia leucopogon, J. L. Gressitt; paratopotypes: 1, same loc., 30003600 m, VI. 1955, Gressitt ; 3, same loc., 3600 m, VI. 1955, Gressitt; 6, same data but VII. 1955 ; 1, same loc., 3450 m, V. 1966, Styphelia suaveolens, Gressitt ; 2, same loc., 3560 m, VIII. 1969, Ericaceae, Gressitt; 1, same loc., 3800 m, VIII. 1969, Gressitt; 7, 3800-4000 m, VIII. 1969, Styphelia douglasii, Gressitt; 1, same data but on Coprosma; 1, same loc., 3560-4000 m, VIII. 1969, Gressitt; 2, same loc., 4000-4200 m, VIII. 1969, Ericaceae, Gressitt; 2, same data but on Hebe; 1, same loc., 4000-4400 m, VIII. 1969, Styphelia, Gressitt; 1, same loc., 4100-4250 m, VIII. 1969, Styphelia, Gressitt; 5, near Lake Aunde [as lower lake], 3400-3500 m, VII. 1963, J. Sedlacek; 4, same loc., 3350 m, VI. 1967, Vaccinium creuentum, G. A. Samuelson; 1, same data but without host; 1, same loc., 3400-3550 m, VI. 1967, Dimorphantheramicrophylla, Samuelson ; 5, same loc., 3560 m, VIII. 1982, Styphelia, Samuelson ; 2, cirque NW of Lake Piunde [as upper lake], 3600-3800 m, VI, 1967, Coprosma papuensis, Samuelson ; 1 , same data but on Eurya; 4, same data but on Styphelia suaveolens; 2, same data but on Symplocos.

Holotype, allotype, many paratypes deposited in BPBM (type no. 13054). Paratypes distributed to ANIC, BMNH, KONE, KU, and NZAC.

REMARKS: The new species differs from all described congeners from New Guinea by having dorsum distinctly granulate instead of smooth-alutaceous ; further differs from dubiosa Jacoby by not having dorsum bicolorous; from gestroi Jacoby by having elytral discal punctures much larger relative to interstices $(0.7-1 \times$ instead of $0.3 \times)$; from viridis Bryant by larger size $(2.5 \mathrm{~mm}$ instead of less than $2 \mathrm{~mm}$ ).

Adults were associated with six families of plant associates, but the larval host is not yet known. Most of the records are from Epacridaceae (Styphelia). The remaining records are probably incidental, with only one to four specimens recorded from each : Ericaceae (Dimorphanthera, Vaccinium), Rubiaceae (Coprosma), Scrophulariaceae (Hebe), Symplocaceae (Symplocos), and Theaceae (Eurya).

\section{Crepidodera stypheliae, new species (Figs. 1H, 2H)}

Male (holotype) . Form subelongate, gently convex along middle, apical region more gradually narrowed, somewhat produced. Dorsum black with dark greenish lustre most pronounced on elytra; antenna with segments 1-4 or- 
ange-testaceous, 5-11 fuscous ; ventral surfaces and femora piceous to black ; tibiae pitchy brown; tarsi paler orange-fuscous. Body length $2.6 \mathrm{~mm}$; breadth 1.35 .

Head: frons carinate medially, depressed laterally ; interantennal space convex medially, shining, briefly impressed laterally, breadth about $1.25 \times$ as broad as transverse diameter of antenna1 socket; orbit about $0.65 \times$ as broad as antenna1 socket; interocular space narrowest above, $1.10 \times$ as broad as greatest diameter of eye ; gena about $0.65 \times$ as deep as eye; postantennal swellings narrow-oblique, raised, smooth, and delimited from vertex by fine oblique groove ; vertex somewhat flattened and alutaceous anteriorly, convex and smooth posteriorly. Antenna extending to middle of elytron ; inner flagellar segments slender, gradually thickened to apices, outer segments becoming more robust and \pm flattened, particularly 10-11. Prothorax $0.75 x$ as long as broad, broadest along middle and distinctly narrower than elytra at humeri ; anterior angle oblique; side feebly convex along middle, straighter basally ; base weakly sinuate, median lobe broadly but weakly produced; disc smoothalutaceous and obscurely punctulate; punctures small, shallow and sometimes $0.2-0.3 \times$ as large as interspaces ; ante-basal impression sinuate, deeply but finely impressed. Elytron about $2.85 \sim$ as long as broad, broadest near middle, preapex obliquely narrowed ; humerus submoderately raised ; disc feebly raised basally, thence shallowly and gradually depressed postbasally ; central discal punctures moderately deep, mostly $0.7-1 \times$ as large as interstices and $1.5-2 \times$ as large as transverse interspaces; interstices feebly raised, slightly convex ; strial punctures obsolescent apically. Ventral surfaces : propleuron smooth, shining; metasternum turgid, smooth-alutaceous, shining medially ; abdominal sterna granulate; sternum 5 with subtriangular depression gradually narrowed and becoming shallower anteriorly, surface with fine median line. Legs :metafemur about 2.2 as long as broad, surface mostly smooth, granulate apically ; lengths of metafemur, -tibia, -tarsus in $\mathrm{mm}$ are $0.92: 0.68: 0.54$; basitarsus not strongly broadened to apex, not quite as long as remainder. Aedeagus( paratype) as figured.

Female (allotype). Similar to male. Apical antenna1 segments less robust than in male; sternum 5 with surface rather evenly convex. Body length 2.5 $\mathrm{mm}$; breadth 1.35 .

Type material: PAPUA NEW GUINEA: Holotype male, allotype female, 10 paratopotypes, Piunde-Aunde Lakes [near lower lake], $3560 \mathrm{~m}, 1$. VIII. 1982, Styphelia, G. A. Samuelson ; paratopotypes : 4, Mt Wilhelm [vicinity of PiundeAunde Lakes], 3600 m, VI. 1955, J. L. Gressitt; 1, same loc., 3000-3600 m, VI. 1955, Gressitt; 1, same loc., 3450 m, V. 1966, Styphelia suaveolens, Gressitt; 4, same loc., 3200-3500 m, VIII. 1969, Styphelia douglasii, Gressitt; 1, same data but on Coprosma; 1, same data but on Compositae, everlasting; 1, same loc., 3500 m, VIII. 1969, tussock, Gressitt; many, same loc., 3560 m, VIII. 1969, Styphelia 
leucopogon, Gressitt ; 6, same 1oc., 3560, 3660 m, VIII. 1969, Ericaceae or Rhododendron, Gressitt; 2, same loc., 3800-4000 m, VIII. 1969, Styphelia douglasii, Gressitt; 1, same loc., $3300 \mathrm{~m}$, VI. 1967, Cyathea atrox, Samuelson; 30, same 1oc., $3200 \mathrm{~m}$, VII. 1982, Styphelia, Samuelson ; 1, same data but on Cyathea atrox; 16, Lake Aunde [as lower lake], 3400-3500 m, VII. 1963, J. Sedlacek; 1, same loc., 34003550 m, VI. 1967, Gaultheria mundula, Samuelson ; 15, same data but $3550 \mathrm{~m} ; 1$, same loc., VIII. 1968, R. C. A. Rice; 2, Lake Piunde [as lower lake], 3560 m, VIII. 1982, J. W. Ismay, Samuelson.

Holotype, allotype, many paratypes deposited in BPBM (type no. 13055). Paratypes distributed to ANIC, BMNH, KONE, KU, and NZAC.

REMARKS: The new species further differs from piundaunde, n. sp. by having apical antenna1 segments more enlarged, pronotal discal punctures obscure, elytral strial punctures obsolescent apically; both of the new species differ from dubiosa Jacoby, gestroi Jacoby, and viridis Bryant by having maximum diameter of eye shorter than breadth of interocular space.

Adults were associated with six families of plant associates, but the larval host is not yet known. Most of the records are from Epacridaceae (Styphelia). The following associations, however, are regarded as incidental ones, as only one to several specimens are recorded from each : Compositae (Gnaphalium), Cyatheaceae (Cyathea tree ferns), Ericaceae (Gaultheria, Rhododendron), Gramineae (tussock grass), and Rubiaceae (Coprosma).

Altitudinal ranges for C. piundaunde and stypheliae are $3350-4100 \mathrm{~m}$ (possibly to $4400 \mathrm{~m}$ ) and $3200-3800 \mathrm{~m}$ (possibly to $4000 \mathrm{~m}$ ) respectively. Both species are common at the lower lake at $3560 \mathrm{~m}$, but stypheliae rarely occurs above $3600 \mathrm{~m}$, while piundaunde commonly occurs above $3600 \mathrm{~m}$. At the lower end of the Piunde-Aunde drainage grassland, ca. $3200-3350 \mathrm{~m}$, stypheliae is common and piundaunde is absent or rare.

\section{Acknowledgements}

I wish to express my gratitude to Professor Y. Hirashima for the privilege of participating in the Papua New Guinea Highlands part of the 1982 Expedition. Special thanks are due to Mr Ben Evara, Director, National Parks Service, Waigani, for logistical help ; Dr Keith Wade, Australian National University, for plant identifications in 1967; Father J. C. E. Riotte, Bishop Museum, Honolulu, for nomenclatorial assistance; and Messrs T. Nagatani, former Bishop Museum artist in Tokyo and Arthur Kodani, present Bishop Museum artist in Honolulu, for preparing the illustrations.

\section{References}

Biass, L. J. 1964. Summary of the sixth Archbold Expedition to New Guinea (1959). Bull. Am. Nat. Hist. 127 : 145-228. 
Central Mapping Bureau. 1974. Papua New Guinea Gazetteer. Department of Lands, Surveys and Mines, Port Moresby.707 p.

Reiner, Ernst. 1960. The glaciation of Mount Wilhelm, Australian New Guinea. Geogr. Rev. 50(4): 491-503.

Royal Australian Survey Corps. 1977. Bundi sheet (No, 7986) Series T601. Papua New Guinea 1: 100,000 Topographic Survey.

Samuelson, G. A. 1971. Alticinae of New Guinea IV (Coleoptera: Chrysomelidae). Pacific Ins $13(3-4): 513-518$.

- 1973. Alticinae of Oceania (Coleoptera : Chrysomelidae). Pacific Ins. Monogr. 30: 1-165.

Wade, L. K. \& D. N. McVean. 1969. Mt Wilhelm Studies I. The alpine and subalpine vegetation. Department of Biogeography and Geomorphology Publ.BG/1. 225 p. Australian National University, Canberra. 